\title{
Nitriles in Heterocyclic Synthesis: Synthesis of Pyrido[3',2':4,5]Thieno[2,3-d] Pyrimidines Derivative
}

\author{
Abdel Haleem Mostafa Hussein, Essam Abdel Raheem Eshak, Fathi Aly Abu-Shanab \\ Chemistry Department, Faculty of Science, Al Azhar University, Assiut, Egypt \\ Email: abdelhaleemmh@yahoo.com
}

Received 10 January 2015; accepted 25 January 2015; published 29 January 2015

Copyright (C) 2015 by authors and OALib.

This work is licensed under the Creative Commons Attribution International License (CC BY). http://creativecommons.org/licenses/by/4.0/

(c) (†) Open Access

\begin{abstract}
6-Amino-3,5-dicyano-4-methylpyridine-2(1H)-thione 1 reacted with $\alpha$-haloketones to give the Salkylated derivatives 2a-m. Compound 2a-m undergoes cyclization into thieno[2,3-d] pyridine derivatives 3a-m upon treatment with ethanolic sodium ethoxide. Saponification of $3 a$ gave the amino acid 4 which afforded 5 when refluxed in $\mathrm{Ac}_{2} \mathrm{O}$. Treatment of 5 with $\mathrm{NH}_{4} \mathrm{OAc} / \mathrm{AcOH}$ gave $6 \mathrm{a}$. Compound 6a also was obtained when $3 \mathrm{c}$ was refluxed in $\mathrm{Ac}_{2} \mathrm{O}$. Reaction of $3 \mathrm{a}$ with formamide gave 7 and with hydrazine hydrate gave 8 . The thiourea derivative 9 was obtained by reaction of 3a with benzoyl isothiocyanate. Compound 9 when refluxed in alcoholic $\mathrm{KOH}$ gave 10 and with $98 \%$ $\mathrm{H}_{2} \mathrm{SO}_{4}$ gave 12. Acetylation of $3 a$ with $\mathrm{Ac}_{2} \mathrm{O}$ gave the acetyl derivative 13 which on treatment with aniline afforded 14. Compound 14 was cyclized with $\mathrm{H}_{2} \mathrm{SO}_{4}$ to 15 . Finally treatment of compound 5 with aniline in $\mathrm{AcOH}$ afforded $6 \mathrm{~b}$.
\end{abstract}

\section{Keywords}

Nitriles, Heterocyclic Synthesis

Subject Areas: Analytical Chemistry, Organic Chemistry

\section{Introduction}

Pyridines are among the most intensively studied heterocyclic compound and their chemistry has been reviewed frequently. Many of the pyridinethiones are biologically active as bactericides [1] [2] evaluated pharmacologically and have been found to show activity against diabetes mellitus, as analagesics and antiinflammants [3]-[6]. On the other hand, pyridothienopyrimidines have been the subject of chemical and biological studies on account of their interesting pharmacological properties. A number of syntheses for substituted derivatives of this triheterocyclic ring system, featuring a variety of pharmacological effects have been developed. Such derivatives have 
analgesic, [7] antipyretic, [8] antianaphilactic, [9] and anti-inflammatory [10] activity. Also, some are clinically effective antialergic [11] or potentially antineophilactic agent [12], and a few possess significant hypocholesteromic [13] activity. These assets promoted us to prepare new pyridothienopyrimidines with potential biological activity. So, it has been found that 6-amino-3,5-dicyano-4-methylpyridine-2(1H)-thione $\mathbf{1}$ [14] reacted with $\alpha$-haloketones and $\alpha$-halonitriles in ethanol and sodium acetate afforded the S-alkylated derivatives 2 . The structure of 2a-m was confirmed by ${ }^{1} \mathrm{H}$ NMR which showed a singlet signal at $\delta 4.0 \mathrm{ppm}$ corresponding to the active methylene group. Compound 2a-m undergoes cyclization into thienopyridine derivatives 3a-m upon treatment with ethanolic sodium ethoxide. The ${ }^{1} \mathrm{H}$ NMR of these compounds revealed the disappearance of the methyl group (Scheme 1).

Saponification of the amino ester $3 \mathbf{a}$ using alcoholic sodium hydroxide gave the sodium salt of the amino acid 4, which afforded 7-amino-2,9-dimethyl-4-oxo-3H-pyrido[3',2':4,5]thieno[3,2-d]oxazine-8-carbonitrile 5 when refluxed in acetic anhydride. Treatment of $\mathbf{5}$ with ammonium acetate in boiling acetic acid led to the formation of thienopyridopyrimidine derivative $\mathbf{6 a}$. Compound $\mathbf{6 a}$ was also obtained by refluxing $\mathbf{3 c}$ in acetic anhydride (Scheme 2).

On the other hand, when 3a was treated with formamide afforded 7-amino-9-methyl-4-oxo-3H-pyrido [3',2': 4,5]thieno[3,2-d]pyrimidine-8-carbonitrile 7. Also 3a was treated with hydrazine hydrate to afford the hydrazide derivative 8. The thiourea derivatives $\mathbf{9}$ was obtained by reaction of 3a with benzoyl isothiocyante [15] [16] in anhydrous acetone solution. Compound $\mathbf{9}$ on alkaline cyclization with alcoholic sodium hydroxide give compound 10 instead of $\mathbf{1 1}$. The ${ }^{1} \mathrm{HNMR}$ data of compound $\mathbf{1 0}$ revealed the absence of aromatic protons and the mass spectrum was compatible with the molecular formula $\mathrm{C}_{11} \mathrm{H}_{7} \mathrm{~N}_{5} \mathrm{OS}_{2}\left(\mathrm{M}^{+}=289\right)$. The cyclic amide structure of compound $\mathbf{1 0}$ furthermore was defined by comparison its cyclic thioester isomer $\mathbf{1 2}$ obtained by ring closure in 98\% sulfuric acid at room temperature [17]-[20]. The two isomeric derivatives $\mathbf{1 0}$ and $\mathbf{1 2}$ are well differentiated according to the alkaline solubility and their ${ }^{1} \mathrm{HNMR}$ and IR spectra. Acetylation of 3a with acetic anhydride gave the acetyl derivatives 13 that on treatment with aniline afforded 14. Compound 14 was cyclized with $98 \%$ sulfuric acid to the pyridothienopyrimidine 15. Treatment of compound $\mathbf{5}$ with aniline in acetic acid afforded compound $\mathbf{6}$. The structure of these compounds was confirmed by ${ }^{1} \mathrm{H}$ NMR, mass, IR spectra and microanalysis (Scheme 3).

\section{Biological Activities}

Most of the synthesized compounds have been tested against four different kinds of bacteria. The result of the antimicrobial studies presented in Table 1. It has been found that the prepared compounds showed antimicrobial<smiles>Cc1c(N)c(C)c(C#N)c(S)c1N</smiles>

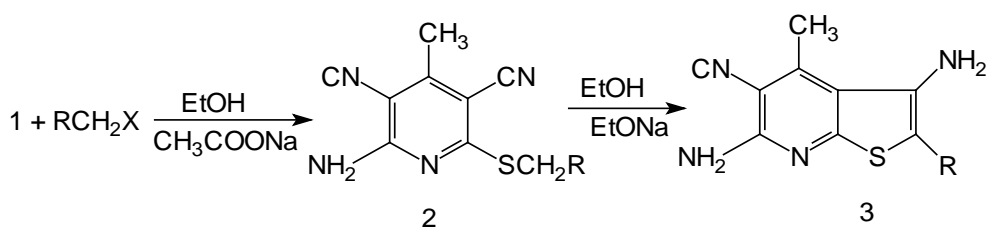

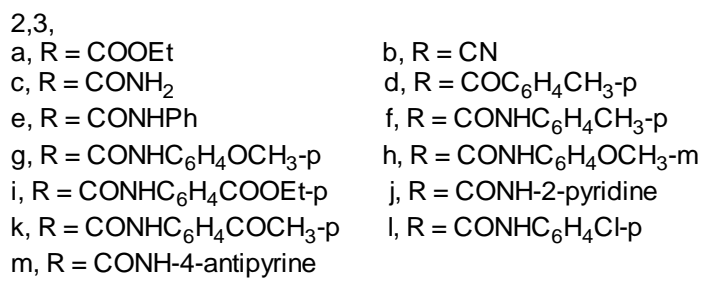

Scheme 1. Synthesis of pyridinethione and thienopyridine. 
<smiles>CCCON1NC(=O)c2sc3nc(N)c(N)c(C)c3c2NC1=S</smiles>

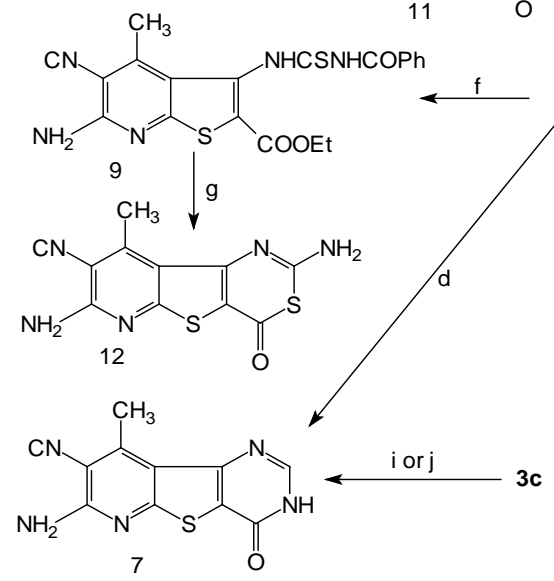

$\mathrm{a}=\mathrm{EtOH} / \mathrm{NaOH}, \mathrm{b}=\mathrm{Ac}_{2} \mathrm{O}, \mathrm{c}=\mathrm{AcOH} / \mathrm{CH}_{3} \mathrm{COONH}_{4}$ $\mathrm{d}=\mathrm{HCONH}_{2}, \mathrm{e}=\mathrm{NH}_{2} \mathrm{NH}_{2} / \mathrm{EtOH}, \mathrm{f}=$ anhydrous aceton/PhCONCS $\mathrm{g}=$ conc. $\mathrm{H}_{2} \mathrm{SO}_{4} \mathrm{~h}=\mathrm{PhNH}_{2} / \mathrm{AcOH} ; \mathrm{i}=\mathrm{HCOOH}, \mathrm{j}=\mathrm{CH}(\mathrm{OEt})_{3} / \mathrm{Ac}_{2} \mathrm{O}$<smiles>Cc1c(C#N)c(N)nc2sc(C(N)=O)c(N)c12</smiles>

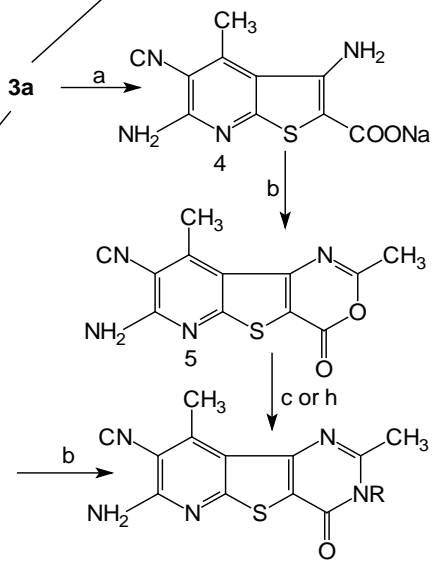

$6 a, b \quad a, R=H$ $\mathrm{b}, \mathrm{R}=\mathrm{Ph}$

Scheme 2. Synthesis of fused pyridine.

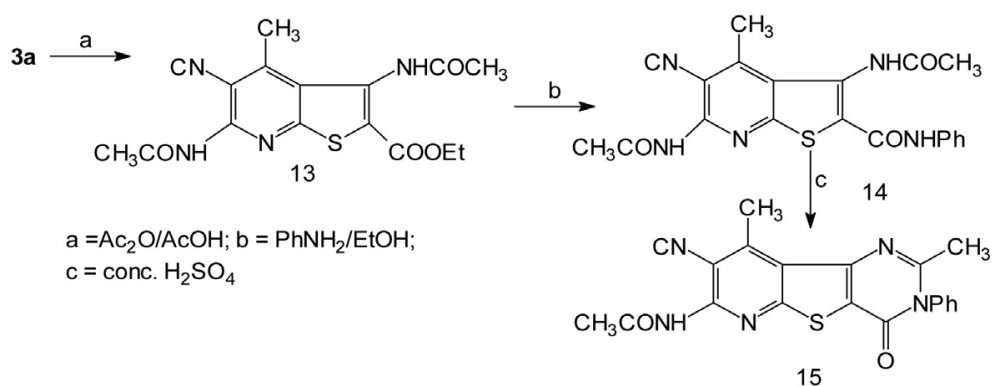

15

Scheme 3. Synthesis of pyridothienoazines.

Table 1. Biological activity of some newly prepared compounds.

\begin{tabular}{|c|c|c|c|c|}
\hline No of Compounds & A & B & C & D \\
\hline 3a & + & + & + & + \\
\hline $\mathbf{3 b}$ & +++ & ++ & + & ++++ \\
\hline $3 c$ & + & ++ & + & +++ \\
\hline 3d & + & +++ & + & ++ \\
\hline $3 \mathbf{e}$ & ++ & +++ & + & ++++ \\
\hline $3 f$ & + & + & + & ++++ \\
\hline $3 g$ & +++ & +++ & + & +++ \\
\hline $3 \mathbf{h}$ & ++++ & ++ & + & ++++ \\
\hline $3 \mathbf{I}$ & + & ++ & + & + \\
\hline $\mathbf{3 j}$ & + & + & + & ++ \\
\hline $3 \mathbf{k}$ & ++ & +++ & ++ & +++ \\
\hline 15 & +++ & ++ & ++++ & +++ \\
\hline
\end{tabular}

Where: A = Staphyllococcus aurous; B = Streptococcus mitor; C = Esherichia coli D = Nisseria sica; $--=$ Negative; + = Poor; + + = Fair; + + + = Good; $++++=$ Very good. 
activity against Staphylococcus aurous, Streptococcus mitor, Esherichia coli and Nisseria sica.

\section{Experimental}

All melting points are uncorrected and were determined on a Gellankamp apparatus, IR spectra were recorded on Schimadzu 470 spectrophotometer in potassium bromide discs; ${ }^{1} \mathrm{H}$ NMR spectra were recorded on a Varian EM-390 (90 Mhz) spectrophotometer using TMS as an internal standard, mass spectrometer MS 30 (AEL) at 70 ev. Analytical data were obtained from the microanalytical data center at Cairo University.

\section{6-Amino-3,5-Dicyano-4-Methylpyridine-2(1h)-Thione 1}

It was prepared according to a liturature procedure [14].

\section{2-Substituted-Mercapto-6-Amino-4-Methylpyridine-3,5-Dicarbonitrile 2a-m. General Procedure}

To a solution of mercaptopyridine $1(0.01 \mathrm{~mol})$ in ethanol $(30 \mathrm{ml})$ and sodium acetate $(0.01 \mathrm{~mol})$, the appropriate halocompound $(0.01 \mathrm{~mol})$ was added. The reaction mixture was refluxed for $1 \mathrm{~h}$. After cooling, the solid product formed was collected by filtration, washed with water several times and recrystallized from the appropriate solvent. The physical data (c.f. Table 2 and Table 3).

\section{3,6-Diamino-4-Methyl-2-Substituted Carboxamidothieno[2,3-b] Pyridine-5-Carbonitrile 3a-m. General Procedure}

To a solution of compound $2(2 \mathrm{~g})$ in absolute ethanol $(30 \mathrm{ml})$, a few drops of sodium ethoxide was added and refluxed for 1 hour. After cooling the solid product formed was collected by filtration and recrystallized from the appropriate solvent.

\section{Sodium-3,6-Diamino-5-Cano-4-Methylthieno[2,3-b] Pyridine-2-Carboxylate 4}

The amino ester 3a was refluxed for $3 \mathrm{~h}$ in ethanolic sodium hydroxide (30 $\mathrm{ml} \mathrm{10 \% ).} \mathrm{The} \mathrm{solid} \mathrm{product} \mathrm{obtained}$ after cooling was collected by filtration, washed was ethanol and left to dry. This compound was used as such in the next procedure.

\section{7-Amino-2,9-Dimethyl-4-0xo-3H-Pyridine[3',2':4,5]Thieno[3,2-d] Oxazine-8-Carbonitrile 5}

The sodium salt $4(0.5 \mathrm{~g})$ was refluxed in acetic anhydride $(30 \mathrm{ml})$ for $3 \mathrm{~h}$. The reaction mixture was left to stand at room temperature and the solid product formed was filtered off and recrystallized from dioxan; $\mathrm{mp} 210^{\circ} \mathrm{C}$; yield 40\%; IR $v \mathrm{~cm}^{-1} 3330-3200\left(\mathrm{NH}_{2}\right), 2190(\mathrm{CN}), 1700$ (CO); MS, $\mathrm{m} / \mathrm{z}=272$; Found: C, 53.0; H, 3.0; N, 20.8; S, 11.77; calcd for $\mathrm{C}_{12} \mathrm{H}_{8} \mathrm{~N}_{4} \mathrm{O}_{2}$ S: C, 52.94; H, 2.96; N, 20.58; S, $11.9 \%$.

\section{Preparation of $6 \mathrm{a}, \mathrm{b}$. General Procedure}

A mixture of oxazine derivative $5(0.01 \mathrm{~mol})$ and ammonium acetate $(0.02 \mathrm{~mol})$ or aniline $(0.01 \mathrm{~mol})$ in acetic acid (30 mol) was heated under reflux for 3 h. The solid product formed after cooling was collected by filtration and recrystallized from the appropriate solvent.

\section{7-Amino-2,9-Dimethyl-4-0xo-3H-Pyrido[3',2':4,5]Thieno[3,2-d] Pyrimidine-8-Carbonitrile 6a}

\subsection{Method A}

Compound 6a was obtained as yellow crystals from DMF/Ethanol; yield 40\%; mp $355^{\circ} \mathrm{C}$; IR $v \mathrm{~cm}^{-1} 3390$ $3225\left(\mathrm{NH}_{2}\right.$ ); 3225 - $3100(\mathrm{NH}) ; 2200(\mathrm{CN}) ; 1651$ (CO); ${ }^{1} \mathrm{H}$ NMR (DMSO-d6) $\delta=2.1$ (s, 3H, $\mathrm{CH}_{3}$ ); 2.3(s, 3H, $\left.\mathrm{CH}_{3}\right) ; 6.4\left(5,2 \mathrm{H}, \mathrm{NH}_{2}\right) ; 12.2(\mathrm{~s}, 1 \mathrm{H}, \mathrm{NH})$; Ms: $\mathrm{m} / \mathrm{z}=271$; Found: $\mathrm{C}, 53.3 ; \mathrm{H}, 3.0 ; \mathrm{N}, 25.9 ; \mathrm{S}, 12.0$; calcd for $\mathrm{C}_{12} \mathrm{H}_{9} \mathrm{~N}_{5} \mathrm{OS}$ : C, 53.13; H, 3.34; N, 25.81; S, 11.82\%. 
Table 2. Physical and analytical data of all newly synthesized compounds 2a-m and 3a-m.

\begin{tabular}{|c|c|c|c|c|c|c|}
\hline \multirow{2}{*}{ No } & \multirow{2}{*}{$\begin{array}{l}. \mathrm{mp}^{\circ} \mathrm{C} \\
\text { Solvent }\end{array}$} & \multirow{2}{*}{$\begin{array}{l}\text { Colour } \\
\text { Yield \% }\end{array}$} & \multirow[t]{2}{*}{ M. Formula MS } & \multicolumn{3}{|c|}{ Calcd/Found } \\
\hline & & & & C & $\mathrm{H}$ & $\mathrm{N}$ \\
\hline $2 \mathrm{a}$ & $\begin{array}{c}170 \\
\text { EtOH }\end{array}$ & $\begin{array}{l}\text { White } \\
70\end{array}$ & $\begin{array}{c}\mathrm{C}_{12} \mathrm{H}_{12} \mathrm{~N}_{4} \mathrm{O}_{2} \mathrm{~S} \\
276\end{array}$ & $\begin{array}{l}52.17 \\
(52.3)\end{array}$ & $\begin{array}{l}4.34 \\
(4.5)\end{array}$ & $\begin{array}{l}17.39 \\
(17.4)\end{array}$ \\
\hline $2 \mathrm{~b}$ & $\begin{array}{c}275 \\
\mathrm{DMF} / \mathrm{EtOH}\end{array}$ & $\begin{array}{c}\text { Green } \\
45\end{array}$ & $\begin{array}{c}\mathrm{C}_{10} \mathrm{H}_{7} \mathrm{~N}_{5} \mathrm{~S} \\
229\end{array}$ & $\begin{array}{l}52.39 \\
(52.5)\end{array}$ & $\begin{array}{l}3.08 \\
(3.2)\end{array}$ & $\begin{array}{l}30.55 \\
(30.8)\end{array}$ \\
\hline $2 c$ & $\begin{array}{l}210 \\
\text { Dioxan }\end{array}$ & $\begin{array}{l}\text { Grey } \\
65\end{array}$ & $\begin{array}{c}\mathrm{C}_{10} \mathrm{H}_{9} \mathrm{~N}_{5} \mathrm{OS} \\
247\end{array}$ & $\begin{array}{l}48.78 \\
(48.9)\end{array}$ & $\begin{array}{l}3.65 \\
(3.7)\end{array}$ & $\begin{array}{l}28.45 \\
(28.5)\end{array}$ \\
\hline $2 \mathrm{~d}$ & $\begin{array}{c}145 \\
\text { EtOH }\end{array}$ & $\begin{array}{l}\text { Yellow } \\
70\end{array}$ & $\begin{array}{c}\mathrm{C}_{17} \mathrm{H}_{14} \mathrm{~N}_{4} \mathrm{OS} \\
322\end{array}$ & $\begin{array}{l}63.35 \\
(63.5)\end{array}$ & $\begin{array}{l}3.54 \\
(3.7)\end{array}$ & $\begin{array}{l}17.39 \\
(17.6)\end{array}$ \\
\hline $2 \mathrm{e}$ & $\begin{array}{c}276 \\
\text { EtOH }\end{array}$ & $\begin{array}{l}\text { Yellow } \\
75\end{array}$ & $\begin{array}{c}\mathrm{C}_{16} \mathrm{H}_{13} \mathrm{~N}_{5} \mathrm{OS} \\
323\end{array}$ & $\begin{array}{l}59.43 \\
(59.6)\end{array}$ & $\begin{array}{l}4.05 \\
(4.3)\end{array}$ & $\begin{array}{l}21.66 \\
(21.9)\end{array}$ \\
\hline $2 f$ & $\begin{array}{c}135 \\
\text { EtOH }\end{array}$ & $\begin{array}{l}\text { White } \\
70\end{array}$ & $\begin{array}{c}\mathrm{C}_{17} \mathrm{H}_{15} \mathrm{~N}_{5} \mathrm{OS} \\
337\end{array}$ & $\begin{array}{l}60.53 \\
(60.7)\end{array}$ & $\begin{array}{l}4.45 \\
(4.7)\end{array}$ & $\begin{array}{l}20.77 \\
(20.9)\end{array}$ \\
\hline $2 g$ & $\begin{array}{c}140 \\
\text { EtOH }\end{array}$ & $\begin{array}{c}\text { Green } \\
68\end{array}$ & $\begin{array}{c}\mathrm{C}_{17} \mathrm{H}_{15} \mathrm{~N}_{5} \mathrm{O}_{2} \mathrm{~S} \\
353\end{array}$ & $\begin{array}{l}57.77 \\
(57.9)\end{array}$ & $\begin{array}{l}4.45 \\
(4.5)\end{array}$ & $\begin{array}{l}19.83 \\
(20.1)\end{array}$ \\
\hline $2 \mathrm{~h}$ & $\begin{array}{c}165 \\
\text { EtOH }\end{array}$ & $\begin{array}{c}\text { Green } \\
60\end{array}$ & $\begin{array}{c}\mathrm{C}_{17} \mathrm{H}_{15} \mathrm{~N}_{5} \mathrm{O}_{2} \mathrm{~S} \\
353\end{array}$ & $\begin{array}{l}57.77 \\
(58.0)\end{array}$ & $\begin{array}{l}4.45 \\
(4.7)\end{array}$ & $\begin{array}{l}19.83 \\
(19.9)\end{array}$ \\
\hline $2 \mathrm{I}$ & $\begin{array}{c}120 \\
\text { EtOH }\end{array}$ & $\begin{array}{l}\text { Black } \\
70\end{array}$ & $\begin{array}{c}\mathrm{C}_{19} \mathrm{H}_{17} \mathrm{~N}_{5} \mathrm{O}_{3} \mathrm{~S} \\
395\end{array}$ & $\begin{array}{l}57.72 \\
(57.9)\end{array}$ & $\begin{array}{l}4.30 \\
(4.6)\end{array}$ & $\begin{array}{l}17.72 \\
(17.9)\end{array}$ \\
\hline $2 \mathrm{j}$ & $\begin{array}{c}320 \\
\text { DMF/EtOH }\end{array}$ & $\begin{array}{l}\text { Grey } \\
75\end{array}$ & $\begin{array}{c}\mathrm{C}_{15} \mathrm{H}_{12} \mathrm{~N}_{6} \mathrm{OS} \\
324\end{array}$ & $\begin{array}{l}55.55 \\
(55.8)\end{array}$ & $\begin{array}{l}3.70 \\
(3.9)\end{array}$ & $\begin{array}{l}26.25 \\
(26.5)\end{array}$ \\
\hline $2 \mathrm{k}$ & $\begin{array}{c}115 \\
\mathrm{MeOH}\end{array}$ & $\begin{array}{l}\text { White } \\
60\end{array}$ & $\begin{array}{c}\mathrm{C}_{18} \mathrm{H}_{15} \mathrm{~N}_{5} \mathrm{O}_{2} \mathrm{~S} \\
365\end{array}$ & $\begin{array}{l}59.17 \\
(59.4)\end{array}$ & $\begin{array}{l}4.14 \\
(4.4)\end{array}$ & $\begin{array}{l}19.17 \\
(19.3)\end{array}$ \\
\hline 21 & $\begin{array}{c}176 \\
\text { EtOH }\end{array}$ & $\begin{array}{c}\text { Green } \\
60\end{array}$ & $\mathrm{C}_{16} \mathrm{H}_{12} \mathrm{~N}_{5} \mathrm{OSCl} 357.5$ & $\begin{array}{l}53.70 \\
(53.9)\end{array}$ & $\begin{array}{l}3.35 \\
(3.5)\end{array}$ & $\begin{array}{l}19.58 \\
(19.8)\end{array}$ \\
\hline $2 \mathrm{~m}$ & $\begin{array}{c}175 \\
\text { EtOH }\end{array}$ & $\begin{array}{c}\text { Yellow } \\
70\end{array}$ & $\begin{array}{c}\mathrm{C}_{21} \mathrm{H}_{19} \mathrm{~N}_{7} \mathrm{O}_{2} \mathrm{~S} \\
433\end{array}$ & $\begin{array}{l}58.19 \\
(58.4)\end{array}$ & $\begin{array}{l}4.42 \\
(4.5)\end{array}$ & $\begin{array}{l}22.62 \\
(22.9)\end{array}$ \\
\hline За & $\begin{array}{c}270 \\
\text { EtOH }\end{array}$ & $\begin{array}{l}\text { Yellow } \\
30\end{array}$ & $\begin{array}{c}\mathrm{C}_{12} \mathrm{H}_{12} \mathrm{~N}_{4} \mathrm{O}_{2} \mathrm{~S} \\
276\end{array}$ & $\begin{array}{l}52.17 \\
(52.4)\end{array}$ & $\begin{array}{l}4.34 \\
(4.4)\end{array}$ & $\begin{array}{l}17.39 \\
(17.5)\end{array}$ \\
\hline $3 b$ & $\begin{array}{c}>360 \\
\text { DMF/EtOH }\end{array}$ & $\begin{array}{c}\text { Black } \\
50\end{array}$ & $\begin{array}{c}\mathrm{C}_{10} \mathrm{H}_{7} \mathrm{~N}_{5} \mathrm{~S} \\
229\end{array}$ & $\begin{array}{l}52.40 \\
(52.6)\end{array}$ & $\begin{array}{l}3.05 \\
(3.3)\end{array}$ & $\begin{array}{l}30.65 \\
(30.9)\end{array}$ \\
\hline 3c & 275 DMF/EtOH & $\begin{array}{c}\text { Orange } \\
50\end{array}$ & $\begin{array}{c}\mathrm{C}_{10} \mathrm{H}_{9} \mathrm{~N}_{5} \mathrm{OS} \\
247\end{array}$ & $\begin{array}{l}48.78 \\
(48.9)\end{array}$ & $\begin{array}{l}3.65 \\
(3.8)\end{array}$ & $\begin{array}{l}28.45 \\
(28.6)\end{array}$ \\
\hline $3 d$ & $\begin{array}{c}200 \\
\text { EtOH }\end{array}$ & $\begin{array}{c}\text { Yellow } \\
65\end{array}$ & $\begin{array}{c}\mathrm{C}_{17} \mathrm{H}_{14} \mathrm{~N}_{4} \mathrm{OS} \\
322\end{array}$ & $\begin{array}{l}63.35 \\
(63.6)\end{array}$ & $\begin{array}{l}3.54 \\
(3.7)\end{array}$ & $\begin{array}{l}17.39 \\
(17.4)\end{array}$ \\
\hline 3e & $\begin{array}{c}310 \\
\text { DMF/EtOH }\end{array}$ & $\begin{array}{c}\text { Yellow } \\
60\end{array}$ & $\begin{array}{c}\mathrm{C}_{16} \mathrm{H}_{12} \mathrm{~N}_{5} \mathrm{OS} \\
323\end{array}$ & $\begin{array}{l}59.44 \\
(59.5)\end{array}$ & $\begin{array}{l}4.02 \\
(4.2)\end{array}$ & $\begin{array}{l}21.16 \\
(21.2)\end{array}$ \\
\hline $3 f$ & $\begin{array}{c}335 \\
\text { DMF/EtOH }\end{array}$ & $\begin{array}{c}\text { Yellow } \\
55\end{array}$ & $\begin{array}{c}\mathrm{C}_{17} \mathrm{H}_{15} \mathrm{~N}_{5} \mathrm{OS} \\
337\end{array}$ & $\begin{array}{l}60.53 \\
(60.7)\end{array}$ & $\begin{array}{l}4.45 \\
(4.5)\end{array}$ & $\begin{array}{l}20.77 \\
(20.9)\end{array}$ \\
\hline $3 g$ & $\begin{array}{c}322 \\
\text { DMF/EtOH }\end{array}$ & $\begin{array}{c}\text { Orange } \\
60\end{array}$ & $\begin{array}{c}\mathrm{C}_{17} \mathrm{H}_{15} \mathrm{~N}_{5} \mathrm{O}_{2} \mathrm{~S} \\
353\end{array}$ & $\begin{array}{l}57.77 \\
(57.8)\end{array}$ & $\begin{array}{l}4.45 \\
(4.7)\end{array}$ & $\begin{array}{l}19.83 \\
(20.0)\end{array}$ \\
\hline $3 \mathrm{~h}$ & $\begin{array}{c}326 \\
\text { DMF/EtOH }\end{array}$ & $\begin{array}{l}\text { Brown } \\
65\end{array}$ & $\begin{array}{c}\mathrm{C}_{17} \mathrm{H}_{15} \mathrm{~N}_{5} \mathrm{O}_{2} \mathrm{~S} \\
353\end{array}$ & $\begin{array}{l}57.77 \\
(57.8)\end{array}$ & $\begin{array}{l}4.45 \\
(4.6)\end{array}$ & $\begin{array}{l}19.83 \\
(20.0)\end{array}$ \\
\hline 3I & $\begin{array}{c}135 \\
\text { EtOH }\end{array}$ & $\begin{array}{l}\text { Brown } \\
30\end{array}$ & $\begin{array}{c}\mathrm{C}_{19} \mathrm{H}_{17} \mathrm{~N}_{5} \mathrm{O}_{3} \mathrm{~S} \\
395\end{array}$ & $\begin{array}{l}57.72 \\
(57.9)\end{array}$ & $\begin{array}{l}3.70 \\
(3.9)\end{array}$ & $\begin{array}{l}26.25 \\
(26.4)\end{array}$ \\
\hline $3 \mathrm{j}$ & $\begin{array}{c}255 \\
\text { dioxan }\end{array}$ & $\begin{array}{c}\text { Grey } \\
25\end{array}$ & $\begin{array}{c}\mathrm{C}_{15} \mathrm{H}_{12} \mathrm{~N}_{6} \mathrm{OS} \\
324\end{array}$ & $\begin{array}{l}55.55 \\
(55.7)\end{array}$ & $\begin{array}{l}3.70 \\
(3.8)\end{array}$ & $\begin{array}{l}26.25 \\
(26.4)\end{array}$ \\
\hline $3 \mathrm{k}$ & $\begin{array}{c}185 \\
\text { EtOH }\end{array}$ & $\begin{array}{l}\text { White } \\
45\end{array}$ & $\begin{array}{c}\mathrm{C}_{18} \mathrm{H}_{17} \mathrm{~N}_{5} \mathrm{O}_{2} \mathrm{~S} \\
355\end{array}$ & $\begin{array}{l}59.17 \\
(59.3)\end{array}$ & $\begin{array}{l}4.10 \\
(4.4)\end{array}$ & $\begin{array}{l}19.17 \\
(19.3)\end{array}$ \\
\hline 31 & $\begin{array}{c}299 \\
\text { dioxan }\end{array}$ & $\begin{array}{c}\text { Yellow } \\
40\end{array}$ & $\begin{array}{c}\mathrm{C}_{16} \mathrm{H}_{12} \mathrm{~N}_{5} \mathrm{OSCl} \\
357.5\end{array}$ & $\begin{array}{l}53.70 \\
(53.9)\end{array}$ & $\begin{array}{l}3.35 \\
(3.4)\end{array}$ & $\begin{array}{l}19.58 \\
(19.7)\end{array}$ \\
\hline $3 \mathrm{~m}$ & $\begin{array}{c}>360 \\
\text { DMF/EtOH }\end{array}$ & $\begin{array}{c}\text { Yellow } \\
65\end{array}$ & $\begin{array}{c}\mathrm{C}_{21} \mathrm{H}_{19} \mathrm{~N}_{9} \mathrm{O}_{2} \mathrm{~S} \\
443\end{array}$ & $\begin{array}{l}54.19 \\
(54.2)\end{array}$ & $\begin{array}{l}4.08 \\
(4.4)\end{array}$ & $\begin{array}{l}21.07 \\
(21.3)\end{array}$ \\
\hline
\end{tabular}




\section{Continued}

\begin{tabular}{|c|c|c|c|c|c|c|}
\hline $3 \mathrm{k}$ & $\begin{array}{c}185 \\
\mathrm{EtOH}\end{array}$ & $\begin{array}{c}\text { White } \\
45\end{array}$ & $\begin{array}{c}\mathrm{C}_{18} \mathrm{H}_{17} \mathrm{~N}_{5} \mathrm{O}_{2} \mathrm{~S} \\
355\end{array}$ & $\begin{array}{l}59.17 \\
(59.3)\end{array}$ & $\begin{array}{l}4.10 \\
(4.4)\end{array}$ & $\begin{array}{l}19.17 \\
(19.3)\end{array}$ \\
\hline 31 & $\begin{array}{c}299 \\
\text { Dioxan }\end{array}$ & $\begin{array}{c}\text { Yellow } \\
40\end{array}$ & $\begin{array}{c}\mathrm{C}_{16} \mathrm{H}_{12} \mathrm{~N}_{5} \mathrm{OSCl} \\
357.5\end{array}$ & $\begin{array}{l}53.70 \\
(53.9)\end{array}$ & $\begin{array}{l}3.35 \\
(3.4)\end{array}$ & $\begin{array}{l}19.58 \\
(19.7)\end{array}$ \\
\hline $3 \mathrm{~m}$ & $\begin{array}{c}>360 \\
\text { DMF/EtOH }\end{array}$ & $\begin{array}{c}\text { Yellow } \\
65\end{array}$ & $\begin{array}{c}\mathrm{C}_{21} \mathrm{H}_{19} \mathrm{~N}_{9} \mathrm{O}_{2} \mathrm{~S} \\
443\end{array}$ & $\begin{array}{l}54.19 \\
(54.2)\end{array}$ & $\begin{array}{l}4.08 \\
(4.4)\end{array}$ & $\begin{array}{l}21.07 \\
(21.3)\end{array}$ \\
\hline
\end{tabular}

Table 3. IR, ${ }^{1} \mathrm{HNMR}$ for the new compounds.

\begin{tabular}{|c|c|c|}
\hline No & IR $v \mathrm{~cm}^{-1}$ & ${ }^{1}$ H NMR $\left.(\delta \text {, DMSO-d })_{6}\right)$ \\
\hline $2 \mathrm{a}$ & 3330 - $3150\left(\mathrm{NH}_{2}\right) ; 2220(\mathrm{CN}) ; 1727$ (ester CO). & $1.1\left(\mathrm{t}, 3 \mathrm{H}, \mathrm{CH}_{3}\right) ; 3.1 ;\left(\mathrm{s}, 3 \mathrm{H}, \mathrm{CH}_{3}\right) ; 4.0$ (q, 2H, $\left.\mathrm{CH}_{2}\right) ; 7.6\left(\mathrm{~s}, 2 \mathrm{H}, \mathrm{NH}_{2}\right)$. \\
\hline $2 b$ & 3415 - $3210\left(\mathrm{NH}_{2}\right) ; 2185(\mathrm{CN})$ & $2.6\left(\mathrm{~s}, 2 \mathrm{H}, \mathrm{CH}_{2}\right) ; 3.1\left(\mathrm{~s}, 3 \mathrm{H}, \mathrm{CH}_{3}\right) ; 6.8\left(\mathrm{~s}, 2 \mathrm{H}, \mathrm{NH}_{2}\right)$. \\
\hline 2c & 3370 - $3160\left(\mathrm{NH}_{2}\right) ; 2190(\mathrm{CN}), 1667$ (CO). & $3.2\left(\mathrm{~s}, 3 \mathrm{H}, \mathrm{CH}_{3}\right) ; 4.0\left(\mathrm{~s}, 2 \mathrm{H}, \mathrm{CH}_{2}\right) ; 5.0\left(\mathrm{~s}, 2 \mathrm{H}, \mathrm{NH}_{2}\right) ; 9.8\left(\mathrm{~s}, 2 \mathrm{H}, \mathrm{NH}_{2}\right)$. \\
\hline $2 \mathrm{~d}$ & 3285 - $3200\left(\mathrm{NH}_{2}\right), 2190(\mathrm{CN}) ; 1660(\mathrm{CO})$. & $\begin{array}{c}2.1\left(\mathrm{~s}, 3 \mathrm{H}, \mathrm{CH}_{3}\right) ; 3.2\left(\mathrm{~s}, 3 \mathrm{H}, \mathrm{CH}_{3}\right) ; 4.0\left(\mathrm{~s}, 2 \mathrm{H}, \mathrm{CH}_{2}\right) ; 6.8-7.8 \\
\left.\text { (m, } 6 \mathrm{H}, \mathrm{Ar}-\mathrm{H} \text { and } \mathrm{NH}_{2}\right) .\end{array}$ \\
\hline $2 \mathrm{e}$ & 3375 - 3165 ( $\left.\mathrm{NH}_{2}-\mathrm{NH}\right) ; 2190$ (CN) 1632 (CO). & $\begin{array}{c}2.1\left(\mathrm{~s}, 3 \mathrm{H}, \mathrm{CH}_{3}\right) ; 3.9\left(\mathrm{~s}, 2 \mathrm{H}, \mathrm{CH}_{2}\right) ; 6.8-7.8\left(\mathrm{~m}, 6 \mathrm{H}, \mathrm{Ar}-\mathrm{H} \text { and } \mathrm{NH}_{2}\right) ; \\
9.7(\mathrm{~s}, 1 \mathrm{H}, \mathrm{NH}) .\end{array}$ \\
\hline $2 \mathrm{f}$ & 3295 - $3140\left(\mathrm{NH}_{2}-\mathrm{NH}\right) ; 2190$ (CN); 1650 (CO). & $\begin{array}{c}2.2\left(\mathrm{~s}, 3 \mathrm{H}, \mathrm{CH}_{3}\right) ; 3.2\left(\mathrm{~s}, 3 \mathrm{H}, \mathrm{H}_{3}\right) ; 4.0\left(\mathrm{~s}, 2 \mathrm{H}, \mathrm{CH}_{2}\right) ; 6.8 \text { - } 7.6(\mathrm{~m}, 4 \mathrm{H}, \mathrm{Ar}-\mathrm{H}) \\
7.8\left(\mathrm{~s}, 2 \mathrm{H}, \mathrm{NH}_{2}\right) ; 9.8(\mathrm{~s}, 1 \mathrm{H}, \mathrm{NH}) .\end{array}$ \\
\hline $2 \mathrm{~g}$ & 3390 - $3200\left(\mathrm{NH}_{2}-\mathrm{NH}\right) ; 2190$ (CN); 1635 (CO). & $\begin{array}{c}2.3\left(\mathrm{~s}, 3 \mathrm{H}, \mathrm{CH}_{3}\right) ; 3.7\left(\mathrm{~s}, 3 \mathrm{H}, \mathrm{OCH}_{3}\right) ; 4.0\left(\mathrm{~s}, 2 \mathrm{H}, \mathrm{CH}_{2}\right) ; 6.8-7.9 \\
\left(\mathrm{~m}, 4 \mathrm{H}, \mathrm{Ar}-\mathrm{H} \text { and } \mathrm{NH}_{2}\right) 10.0(\mathrm{~s}, 1 \mathrm{H}, \mathrm{NH}) .\end{array}$ \\
\hline $2 \mathrm{~h}$ & 3295 - $3170\left(\mathrm{NH}_{2}\right) ; 2195(\mathrm{CN}) ; 1642(\mathrm{CO})$. & $\begin{array}{l}2.0\left(\mathrm{~s}, 3 \mathrm{H}, \mathrm{CH}_{3}\right) ; 3.7\left(\mathrm{~s}, 3 \mathrm{H}, \mathrm{OCH}_{3}\right) ; 4.0\left(\mathrm{~s}, 2 \mathrm{H}, \mathrm{CH}_{2}\right) ; \\
\quad 7.0-7.9\left(\mathrm{~m}, 4 \mathrm{H}, \mathrm{Ar}-\mathrm{H} \text { and } \mathrm{NH}_{2}\right) 10.2(\mathrm{~s}, 1 \mathrm{H}, \mathrm{NH})\end{array}$ \\
\hline $2 \mathrm{i}$ & $\begin{array}{c}3400-3210\left(\mathrm{NH}_{2}-\mathrm{NH}\right) ; 2195(\mathrm{CN}), 1690 \text { (ester } \\
\text { CO); } 1630 \text { (CO). }\end{array}$ & $\begin{array}{c}1.1\left(\mathrm{t}, 3 \mathrm{H}, \mathrm{CH}_{3}\right) ; 2.3\left(\mathrm{~s}, 3 \mathrm{H}, \mathrm{CH}_{3}\right) ; 4.0\left(\mathrm{~s}, 2 \mathrm{H}, \mathrm{CH}_{2}\right) ; \\
4.2\left(\mathrm{q}, 2 \mathrm{H}, \mathrm{CH}_{2}\right) ; 6.8-7.9\left(\mathrm{~m}, 4 \mathrm{H}, \mathrm{Ar}-\mathrm{H} \text { and } \mathrm{NH}_{2}\right) ; 9.5(\mathrm{~s}, 1 \mathrm{H}, \mathrm{NH}) .\end{array}$ \\
\hline $2 \mathrm{j}$ & 3450 - $3110\left(\mathrm{NH}_{2}-\mathrm{NH}\right) ; 2195(\mathrm{CN}) ; 1640(\mathrm{CO})$ & $\begin{array}{c}3.4\left(\mathrm{~s}, 3 \mathrm{H}, \mathrm{CH}_{3}\right) ; 4.8\left(\mathrm{~s}, 3 \mathrm{H}, \mathrm{CH}_{3}\right) ; 4.0\left(\mathrm{~s}, 2 \mathrm{H}, \mathrm{CH}_{2}\right) \\
7.2 \text { - } 7.9\left(\mathrm{~m}, 6 \mathrm{H} \text {, pyrdine- } \mathrm{H} \text { and } \mathrm{NH}_{2}\right) ; 9.9(\mathrm{~s}, 1 \mathrm{H}, \mathrm{NH}) \text {. }\end{array}$ \\
\hline $2 \mathrm{k}$ & $\begin{array}{c}3400 \text { - } 3250\left(\mathrm{NH}_{2}-\mathrm{NH}\right) ; 2200(\mathrm{CN}) ; 1674(\mathrm{CO}) \\
1635 \text { (CO). }\end{array}$ & $\begin{array}{l}2.3\left(\mathrm{~s}, 3 \mathrm{H}, \mathrm{CH}_{3}\right) ; 3.2\left(\mathrm{~s}, 3 \mathrm{H}, \mathrm{CH}_{3}\right) ; 4.0\left(\mathrm{~s}, 2 \mathrm{H}, \mathrm{CH}_{2}\right) ; \\
6.8-7.9\left(\mathrm{~m}, 4 \mathrm{H}, \mathrm{Ar}-\mathrm{H} \text { and } \mathrm{NH}_{2}\right) 10.0(\mathrm{~s}, 1 \mathrm{H}, \mathrm{NH}) .\end{array}$ \\
\hline 21 & 3455 - $3125\left(\mathrm{NH}_{2}-\mathrm{NH}\right) ; 2190$ (CN), 1634 (CO). & $\begin{array}{c}2.3\left(\mathrm{~s}, 3 \mathrm{H}, \mathrm{CH}_{3}\right) ; 3.9\left(\mathrm{~s}, 2 \mathrm{H}, \mathrm{CH}_{2}\right) ; \\
6.8-7.9\left(\mathrm{~m}, 4 \mathrm{H}, \mathrm{Ar}-\mathrm{H} \text { and } \mathrm{NH}_{2}\right) 9.8(\mathrm{~s}, 1 \mathrm{H}, \mathrm{NH}) .\end{array}$ \\
\hline $2 \mathrm{~m}$ & 3370 - 3270 (NH2-NH); 2195 (CN); 1637 (CO). & \\
\hline За & 3400 - $3145\left(\mathrm{NH}_{2}\right) ; 2190$ (CN); 1727 (ester CO). & $\begin{array}{c}1.2\left(\mathrm{t}, 3 \mathrm{H}, \mathrm{CH}_{3}\right) ; 2.3\left(\mathrm{~s}, 3 \mathrm{H}, \mathrm{CH}_{3}\right) ; 4.0\left(\mathrm{q}, 2 \mathrm{H}, \mathrm{CH}_{2}\right) ; \\
3.5\left(\mathrm{~s}, 2 \mathrm{H}, \mathrm{NH}_{2}\right) ; 7.9\left(\mathrm{~s}, 2 \mathrm{H}, \mathrm{NH}_{2}\right)\end{array}$ \\
\hline $3 b$ & 3260 - $3160\left(\mathrm{NH}_{2}\right) ; 2190(\mathrm{CN})$. & $2.2\left(\mathrm{~s}, 3 \mathrm{H}, \mathrm{CH}_{3}\right) ; 3.6$ - $4.0\left(\mathrm{br}, 4 \mathrm{H}, 2 \mathrm{NH}_{2}\right)$. \\
\hline 3C & 3440 - $3155\left(\mathrm{NH}_{2}\right) ; 2190$ (CN); 1649 (CO). & 2.8 (s, 3H, $\left.\mathrm{CH}_{3}\right) ; 3.2\left(\mathrm{~s}, 2 \mathrm{H}, \mathrm{NH}_{2}\right) ; 5.5$ (br, 4H, $\left.2 \mathrm{NH}_{2}\right)$. \\
\hline 3d & 3350 - $3175\left(\mathrm{NH}_{2}\right), 2190(\mathrm{CN}) ; 1660(\mathrm{CO})$. & $2.3\left(\mathrm{~s}, 3 \mathrm{H}, \mathrm{CH}_{3}\right) ; 3.8\left(\mathrm{~s}, 3 \mathrm{H}, \mathrm{CH}_{3}\right) ; 7$ - 8 (m, 8H, Ar-H and $\left.2 \mathrm{NH}_{2}\right)$. \\
\hline $3 f$ & 3395 - $3095\left(\mathrm{NH}_{2}-\mathrm{NH}\right) ; 2210$ (CN); 1646 (CO). & $\begin{array}{c}2.2\left(\mathrm{~s}, 3 \mathrm{H}, \mathrm{CH}_{3}\right) ; 3.1\left(\mathrm{~s}, 3 \mathrm{H}, \mathrm{CH}_{3}\right) ; 3.5\left(\mathrm{~b}, 4 \mathrm{H}, \mathrm{NH}_{2}\right) ; \\
6.8-7.5\left(\mathrm{~m}, 7 \mathrm{H}, \mathrm{Ar}-\mathrm{H} \text { and } \mathrm{NH}_{2}\right) ; 9.0(\mathrm{~s}, 1 \mathrm{H}, \mathrm{NH}) .\end{array}$ \\
\hline $3 g$ & 3485 - $3100\left(\mathrm{NH}_{2}-\mathrm{NH}\right) ; 2215(\mathrm{CN}) ; 1682(\mathrm{CO})$ & $\begin{array}{l}2.3\left(\mathrm{~s}, 3 \mathrm{H}, \mathrm{CH}_{3}\right) ; 3.4\left(\mathrm{~b}, 4 \mathrm{H}, \mathrm{NH}_{2}\right) ; 3.8\left(\mathrm{~s}, 3 \mathrm{H}, \mathrm{OCH}_{3}\right) \\
\quad 7.0-7.5\left(\mathrm{~m}, 7 \mathrm{H}, \mathrm{Ar}-\mathrm{H} \text { and } \mathrm{NH}_{2}\right) ; 9.2(\mathrm{~s}, 1 \mathrm{H}, \mathrm{NH})\end{array}$ \\
\hline $3 \mathrm{i}$ & $\begin{array}{l}3395 \text { - } 3190\left(\mathrm{NH}_{2}-\mathrm{NH}\right) ; 190(\mathrm{CN}) \\
\quad 1700 \text { (ester CO); } 1680 \text { (CO). }\end{array}$ & $\begin{array}{c}1.2\left(\mathrm{t}, 3 \mathrm{H}, \mathrm{CH}_{3}\right) ; 2.3\left(\mathrm{~s}, 3 \mathrm{H}, \mathrm{H}_{3}\right) ; 3.5\left(\mathrm{~b}, 4 \mathrm{H}, 2 \mathrm{CH}_{2}\right) ; 4.0\left(\mathrm{q}, 2 \mathrm{H}, \mathrm{CH}_{2}\right) ; \\
7.5-8.0\left(\mathrm{~m}, 8 \mathrm{H}, \mathrm{Ar}-\mathrm{H} \text { and } 2 \mathrm{NH}_{2}\right) ; 10.4(\mathrm{~s}, 1 \mathrm{H}, \mathrm{NH}) .\end{array}$ \\
\hline $3 \mathrm{j}$ & 3450 - $3185\left(\mathrm{NH}_{2}, \mathrm{NH}\right) ; 2210(\mathrm{CN}) ; 1650(\mathrm{CO})$ & $3.2\left(\mathrm{~s}, 3 \mathrm{H}, \mathrm{CH}_{3}\right) ; 7.0$ - $7.6(\mathrm{~m}, 4 \mathrm{H}, \mathrm{Ar}-\mathrm{H}) ; 8.0\left(\mathrm{~s}, 4 \mathrm{H}, 2 \mathrm{NH}_{2}\right) ; 10.0(\mathrm{~s}, 2 \mathrm{H}, \mathrm{NH})$. \\
\hline $3 \mathrm{k}$ & 3390 - $3185\left(\mathrm{NH}_{2}, \mathrm{NH}\right) ; 2195$ (CN); 1650 (CO). & $\begin{array}{c}3.0\left(\mathrm{~s}, 3 \mathrm{H}, \mathrm{CH}_{3}\right) ; 3.3\left(\mathrm{~s}, 3 \mathrm{H}, \mathrm{CH}_{3}\right) ; 7.1-7.6(\mathrm{~m}, 4 \mathrm{H}, \mathrm{Ar}-\mathrm{H}) ; \\
8.0\left(\mathrm{~s}, 4 \mathrm{H}, 2 \mathrm{NH}_{2}\right) ; 10.9(\mathrm{~s}, 2 \mathrm{H}, \mathrm{NH})\end{array}$ \\
\hline 31 & 3430 - 3331(NH2-NH); 2190 (CN); 1641 (CO). & $2.9\left(\mathrm{~s}, 3 \mathrm{H}, \mathrm{CH}_{3}\right) ; 6.8$ - $8.0\left(\mathrm{~m}, 8 \mathrm{H}, \mathrm{Ar}-\mathrm{H}\right.$ and $\left.2 \mathrm{NH}_{2}\right) ; 9.0(\mathrm{~s}, 1 \mathrm{H}, \mathrm{NH})$. \\
\hline $3 \mathrm{~m}$ & 3390 - $3015\left(\mathrm{NH}_{2}-\mathrm{NH}\right) ; 2205$ (CN); 1657 (CO). & \\
\hline
\end{tabular}

\subsection{Method B for Preparation of 6a}

A solution of 3c $(0.01 \mathrm{~mol})$ in acetic anhydride $(20 \mathrm{~mol})$ was heated under refluxe for $5 \mathrm{~h}$. The solid product so formed after cooling was filtered off and recrystallized from DM/Ethanol as yellow crystals; yield 45\%; $\mathrm{m} \mathrm{p}$ and mixed $\mathrm{m} p$ as $6 \mathrm{a}$. 


\section{7-Amino-2,9-Dimethyl-4-0X0-3-Phenylpyrido[3',2':4,5] Thieno[3,2-d]} Pyrimidine-8-Carbonitrile (6b)

Compound $\mathbf{6 b}$ was crystallized from DMF/Dioxan as yellow crystals; yield 45\%; mp > 360 ${ }^{\circ}$; IR $v \mathrm{~cm}^{-1} 3355$ $3220\left(\mathrm{NH}_{2}\right)$; 3220 - $3150(\mathrm{NH}) ; 2200(\mathrm{CN}) ; 1655$ (CO); Found: C, 62.5; H, 3.9: N, 20.3; calcd for $\mathrm{C}_{18} \mathrm{H}_{13} \mathrm{~N}_{5} \mathrm{OS}$ : C, 62.23; H, 3.77; N, 20.16\%.

\section{7-Amino-9-Methyl-4-0xo-3H-Pyrido[3',2':4,5]Thieno[3,2-d] Pyrimidine-8-Carbonitrile 7}

\subsection{Method A}

A solution of 3a $(0.01 \mathrm{~mol})$ in formamide $(10 \mathrm{~mol})$ was heated under reflux for $2 \mathrm{~h}$. The reaction mixture was poured on ice water. The solid product formed was filtered off, washed with water several times, dried and recrystallized from ethanol as red crystals; yield 37\%; mp 230 ${ }^{\circ} \mathrm{C}$; IR $v \mathrm{~cm}^{-1} 33703220\left(\mathrm{NH}_{2}\right)$; 3220 - $3165(\mathrm{NH})$; 2190 (CN); 1663 (CO) MS: $\mathrm{m} / \mathrm{z}=257$; Found: C, 51.5; H, 2.8; N, 27.6; S, 12.7; calcd for $\mathrm{C}_{12} \mathrm{H}_{7} \mathrm{~N}_{5} \mathrm{OS}$ : 51.36; $\mathrm{H}$, 2.72; N, 27.73; S, 12.46\%.

\subsection{Method B}

A suspension of 3c $(0.01 \mathrm{~mol})$ and triethylorthoformete $(3 \mathrm{~mol})$ in acetic anhydride $(30 \mathrm{ml})$ was refluxed for $3 \mathrm{~h}$. The reaction mixture was poured on water and left to stand overnight. The solid precipitate formed was filtered off and recrystallized from ethanol as red crystals; yield $40 \%$; $\mathrm{mp}$ and mixed mp as 7 .

\subsection{Method C}

Compound 3c (2 g) was dissolved in formic acid $(20 \mathrm{ml})$ and heated under refluxe for $3 \mathrm{~h}$. The solid product thus formed on cooling was collected by filtration and recrystallized from ethanol as red crystals; yield $41 \%$; mp and mixed mp as 7.

\section{3,6-Diamino-2-Carbohydrazido-4-Methylthieno[2,3-b] Pyridine-5-Carbonitrile 8}

To a solution of $3 \mathbf{a}(0.01 \mathrm{~mol})$ in ethanol $(30 \mathrm{ml})$, the hydrazine hydrate $(0.02 \mathrm{~mol})$ was added. The reaction mixture was refluxed for $3 \mathrm{~h}$. The solid product formed was collected by filtration and recrystallized from DMF/Dioxan as white crystals; yield 66\%; mp $295^{\circ} \mathrm{C}$; IR $v \mathrm{~cm}^{-1} 3400-3220\left(\mathrm{NH}_{2}\right)$; $3220-3100(\mathrm{NH}) ; 2195$ (CN); 1650 (CO); MS: $\mathrm{m} / \mathrm{z}=262$; Found: $\mathrm{C}, 45.9 ; \mathrm{H}, 3.7 ; \mathrm{N}, 32.5 ; \mathrm{S}, 12.4$; calcd for $\mathrm{C}_{10} \mathrm{H}_{10} \mathrm{~N}_{6} \mathrm{OS}$ : C, 54.79; $\mathrm{H}$, $3.84 ; \mathrm{N}, 32.04 ; \mathrm{S}, 12.22 \%$.

\section{Ethyl-2-Amino-3-Cyano-4-Methyl-5-(Benzoylthiourea)Thieno[2,3-b] Pyridine-6-CArboxylate 9}

To a solution of 3a in anhydrous acetone, benzoyl isothiocyanate (prepared in situ by refluxed mixture of benzoyl chloride $(0.1 \mathrm{~mol})$ and ammonium thiocyanate $(0.1 \mathrm{~mol})$ in anhydrous acetone for ten minutes) was added. The reaction mixture was refluxed for 3 hours, then poured onto cold water. The precipitate was collected by filtration, repeatedly washed with cold water and recrystallized from ethanol as orange crystals; yield $50 \% \mathrm{mp}$ $145^{\circ} \mathrm{C}-150^{\circ} \mathrm{C}$; IR $v \mathrm{~cm}^{-1} 3340$ - $3200\left(\mathrm{NH}_{2}-\mathrm{NH}\right) ; 2190(\mathrm{CN}) ; 1780$ (CO) ester; $1650(\mathrm{CO})$; MS: $\mathrm{m} / \mathrm{z}=439$; Found: C, 55.9; H, 3.9; H, 15.0; S, 15.1; calcd for $\mathrm{C}_{20} \mathrm{H}_{17} \mathrm{~N}_{5} \mathrm{O}_{3} \mathrm{~S}_{2}$ : C, 54.66; H, 3.87; N, 15.94; S, 14.59\%.

\section{7-Amino-9-Methyl-8-Cyano-4-0xo-1,2,3,4-Tetrahydropyrido $\left[3^{\prime}, 2\right.$ ':4,5] Thieno[3,2-d]Pyrimidine-2-Thiol 10}

A sample of compound 9 ( $1 \mathrm{~g})$ was dissolved in $2 \mathrm{~N}$ ethanolic sodium hydroxid solution (30 $\mathrm{ml})$ and refluxed for $6 \mathrm{~h}$. The reaction mixture was poured onto ice/water and acidified with $10 \% \mathrm{HCl}$. The solid formed was collected by filtration and recrystallized from DMF/water as brown crystals; yield $66 \%$; mp $>350^{\circ} \mathrm{C}$; IR $v \mathrm{~cm}^{-1}$ 3300 - $3200\left(\mathrm{NH}_{2}\right) ; 2200(\mathrm{CN}) ; 1640$ (CO); MS: $\mathrm{m} / \mathrm{z}=$ 289; Found: C, 45.8; H, 2.5; N, 25.0; s, 22.4; calcd for $\mathrm{C}_{11} \mathrm{H}_{7} \mathrm{~N}_{5} \mathrm{OS}_{2}$ : C, 45.67; H, 2.42; N, 24.22; 22.16\%. 


\section{Preparation of Compounds 12 and 15. General Procedure}

A solution of compound 9 or 14 ( $1 \mathrm{~g})$ in $98 \%$ sulfuric acid $(5 \mathrm{ml})$ was stirred $1 \mathrm{~h}$. then left at room temperature for 5 days. The solid product formed after pouring the clear solution in ice water $(100 \mathrm{ml})$ was collected, wash with water, dried and recrystalized from the appropriate solvents.

\section{2,7-Diamino-9-Methyl-8-Cyano-4-0xo-2,3,4-Trihydropyrido} $\left[3^{\prime}, 2^{\prime}, 4,5\right]$ Thieno[2,3-d] Thiazine 12

Compound 12 was obtained as brown crystals from DMF/water; $\mathrm{mp}>350^{\circ} \mathrm{C}$; yield $30 \%$; IR $v \mathrm{~cm}^{-1} 3375$ - 3270 $\left(\mathrm{NH}_{2}\right), 2220(\mathrm{CN}), 1662$ (CO); Found: C, 45.9; H, 2.6; N, 24.4; calcd for $\mathrm{C}_{11} \mathrm{H}_{7} \mathrm{~N}_{5} \mathrm{OS}_{2}$ : C, 45.67; H, 2.42; N, $24.22 \%$.

\section{2,5-Diacetylamino-3-Cyano-4-Methylthieno[2,3-b]Pyridine-6-Carboxylate 13}

To a solution of compound $9(0.01 \mathrm{~mol})$ in acetic acid $(30 \mathrm{ml})$, the appropriate of acetic anhydride $(3 \mathrm{ml})$ was added. The reaction mixture was heated under reflux for $3 \mathrm{~h}$. The solid product formed after cooling was collected by filtration and recrystallization from methanol as orange crystal. $\mathrm{mp} 330^{\circ} \mathrm{C}$; yield $60 \%$; IR $v \mathrm{~cm}^{-1} 3330$ 3150 (2NH), 2200 (CN), 1724 (CO ester), 1641(CO)); ${ }^{1} \mathrm{H}$ NMR (DMSO-d6) $\delta=1.4$ (s, 3H, $\mathrm{CH}_{3}$ ); 2.4 (s, 3H, $\mathrm{CH}_{3}$ ); 2.4 (br, $6 \mathrm{H}, 2 \mathrm{CH}_{3}$ ); 4.4 (q, 2H, $\mathrm{CH}_{2}$ ); 8.4 (br, 2H, 2NH); Found: C, 53.5; H, 4.8; N, 15.8; S, 9.2; calcd for $\mathrm{C}_{16} \mathrm{H}_{16} \mathrm{~N}_{4} \mathrm{O}_{4} \mathrm{~S}$ : C, 53.32; H, 4.47; N, 15.55; S, 8.90\%.

\section{2,5-Diacetylamino-3-Cyano-4-Methylthieno[2,3-b]Pyridine-6-Benzanilide 14}

To a solution of compound $13(0.01 \mathrm{~mol})$ in ethanol $(30 \mathrm{ml})$ the appropriate of aniline $(0.01 \mathrm{~mol})$ was added, the reaction mixture was heated under reflux for $3 \mathrm{~h}$. The solid product formed after cooling was collected by filtration and recrystallized from acetic acid as yellow crystals; $\mathrm{mp}>350^{\circ} \mathrm{C}$; yield $50 \%$; IR $v \mathrm{~cm}^{-1} 3450-3195(\mathrm{NH})$, 2220 (CN), 1670 (CO); ${ }^{1} \mathrm{H}$ NMR (DMSO-d6) $\delta=2.3$ (s, 3H, $\mathrm{CH}_{3}$ ); 2.8 (d, 6H, 2CH $)$; 8.2 (s, $1 \mathrm{H}, \mathrm{NH}$ ); 4.2 - 4.6 (m, 5H, Ar-H); 8.4 (br, $1 \mathrm{H}, \mathrm{NH}) ; 10.4$ (s, $1 \mathrm{H}, \mathrm{NH})$; MS: $\mathrm{m} / \mathrm{z}=$ 407; Found: 86.1; H, 4.4; N, 17.4: S, 8.0; calcd for $\mathrm{C}_{20} \mathrm{H}_{17} \mathrm{~N}_{5} \mathrm{O}_{3} \mathrm{~S}$ : C, 85.96; $\mathrm{H}, 4.21 ; \mathrm{N}, 17.19 ; \mathrm{S}, 7.87 \%$.

\section{7-Acetylamino-2,9-Dimethyl-3-Phenyl-4-0xo-Pyrido[3',2',4, 5] Thieno[3,2-d] Pyrimidine-8-Carbonitrile 15}

Compound 15 was obtained as yellow crystals from dioxan; $\mathrm{mp}>350^{\circ} \mathrm{C}$; yield $30 \%$; IR $v \mathrm{~cm}^{-1} 3330(\mathrm{NH}), 2210$ (CN), 1693 (2CO); MS: $\mathrm{m} / \mathrm{z}=389$; Found: 61.9; $\mathrm{H}, 3.9 ; \mathrm{N}, 18.1$; S, 18.3; calcd for $\mathrm{C}_{20} \mathrm{H}_{15} \mathrm{~N}_{5} \mathrm{O}_{2} \mathrm{~S}$ : C, 61.68; $\mathrm{H}$, 3.88 ; N, 17.98; S, 8.23\%.

\section{Biological Testing}

The newly synthesized compounds were dissolved in propylene glycol $(10 \mathrm{mg} / 20 \mathrm{ml})$ and transferred to a filter paper disc $(10 \mathrm{~mm})$ diffusion plate method [18]. The bacterial suspension was prepared by adding $20 \mathrm{ml}$ of distilled water to 10-d-old cultures of the test bacteria grown on a nutrient agar of NA. The spore suspension was prepared by adding $20 \mathrm{ml}$ of distilled water to 10 -d-od cultures of the test bacteria.

\section{References}

[1] Göbel, W. (1974) Pharmazie, 29, 744.

[2] Black, J.G. and Howes, D. (1979) Toxicology Annual, 3, 1.

[3] Nakanish, M., Imamura, H., Marayama, Y. and Hirosuki (1970) Chem. Abst, 90, 272. (1970) Chem. Abst, 90, 54504.

[4] Nakanish, M., Imamura, H., Marayama, Y. and Goto, K. (1970) Yakujaka. Zasshi, 90, 548.

[5] Nakanish, M., Imamura, H., Marayama, Y. and Goto, K. (1970) Yakujaka. Zasshi, 73, 4378.

[6] Nakanish, M., Imamura, H., Marayama, Y. and Marayama, Y. (1970) Arzneim. Forsh, 20, 4378.

[7] Dave, C.G., Shah, P.R., Dave, K.C. and Patel, V.J. (1989) Journals of the Indian Chemical Society, 66, 48.

[8] (a) Bousquet, E., Romero, G., Guerrera, F., Caruso, A. and Roxas, M.A. (1985) Farmaco Ed. Sci., 40, 869. 
(b) Bousquet, E., Guerrera, F., Siracusa, A., Caruso, A. and Roxas, M.A. (1984) Farmaco Ed. Sci., 39, 110.

[9] (a) Vieweg, H., Leistner, S., Wagner, G., Boehm, N., U. Krasset R., Lohmann, G.D. and Loban, G. (1988) East German Patent No. 257,803. Chem. Abstr., 110, 95262 p (1989).

(b) Vieweg, H., Leistner, S., Wagner, G., Boehm, N., U. Krasset R., Lohmann, G.D. and Loban, G. (1988) East German Patent No. 258,234. Chem. Abstr., 110, 95263 p (1989).

[10] (a) Leistner, S., Wagner, G., Guetecharo, M. and Glusa, E. (1986) Pharmazie, 41, 54.

(b) Radinovskaya, L.A. and Sharamin, A. (1988) Khim. Geterotsikl. Soedin, 805 and References Therein.

(c) Chaykovsky, M., Lin, M., Rosowsky, A. and Modest, E.J. (1973) 2,4-Diaminothieno[2,3-d]pyrimidines as Antifolates and Antimalarials. 2. Synthesis of 2,4-Diaminopyrido[4',3':4,5]thieno[2,3-d]pyrimidines and 2,4-Diamino-8H-thiopyrano[4',3':4,5]thieno[2,3-d]pyrimidines. Journal of Medicinal Chemistry, 10, 188-191.

http://dx.doi.org/10.1021/jm00261a003

(d) Eslager, E.F., Jacob, P.W. and Leslic, M. (1972) Journal of Heterocyclic Chemistry, 9, 775.

[11] Madding, G.D. and Thompson, M.D. (1987) Regioselective Syntheses of 2-Amino-4,5-dialkylthiophene-3-carboxylates and Their Conversion to 3,4-Dihydro-4-oxothieno[2,3-d]pyrimidine-2-carboxylates. Journal of Heterocyclic Chemistry, 24, 581-587.

[12] Cheng, C.C. (1989) In: Ellis, G.P. and West, G.B., Eds., Progress in Medical Chemistry, Vol. 25, Elsevier Science Publishers, Amsterdam, 35.

[13] Sishoo, C.J., Devani, M.B. and Bhadti, V.S. (1983) Indian Patent No. 151,456. Chem. Abstr., 100, 209858 (1993).

[14] Elnagdi, M.H. and Erian, A.W. (1995) Bull. Soc Chem Fr, 132, 920.

[15] Frank, R.I. and Smith, P.V. (1948) In: Snyder, H.R., Ed., Org. Synth., Vol. 28, John Wiley \& Sons, Inc., New York, 89.

[16] Douglass, I.B. and Dains, F.B. (1934) The Preparation and Hydrolysis of Mono- and Disubstituted Benzoylthioureas. Journal of the American Chemical Society, 56, 1408-1409. http://dx.doi.org/10.1021/ja01321a061

[17] Errede, L.A., Oien, H.T. and Yarian, D.R. (1977) Journal of Organic Chemistry, 42, 1.

[18] Eisenaecher, T., Pech, R. and Böhm, R. (1991) Über neue Pyrazolverbindungen. IV Darstellung und Cyclisierungsverhalten einiger akzeptorsubstituierter N-(Pyrazol-3-yl)-thioharnstoffe. Journal für Praktische Chemie, 333, 437-446. http://dx.doi.org/10.1002/prac.19913330309

[19] Black, J.G. and Howes, D. (1979) Toxieology Annual, 3, 1.

[20] Loo, Y.H., Shell, P.S., Ehruch, T., Mecuries, J., Savage, G.M. and Sylvester, J.C. (1945) J. Bacterial, 50, 701. 
Scientific Research Publishing (SCIRP) is one of the largest Open Access journal publishers. It is currently publishing more than 200 open access, online, peer-reviewed journals covering a wide range of academic disciplines. SCIRP serves the worldwide academic communities and contributes to the progress and application of science with its publication.

Other selected journals from SCIRP are listed as below. Submit your manuscript to us via either submit@scirp.org or Online Submission Portal.
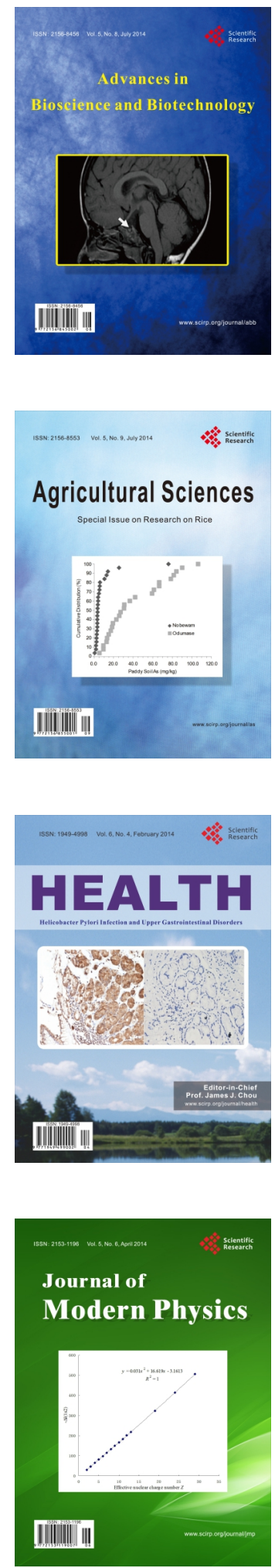
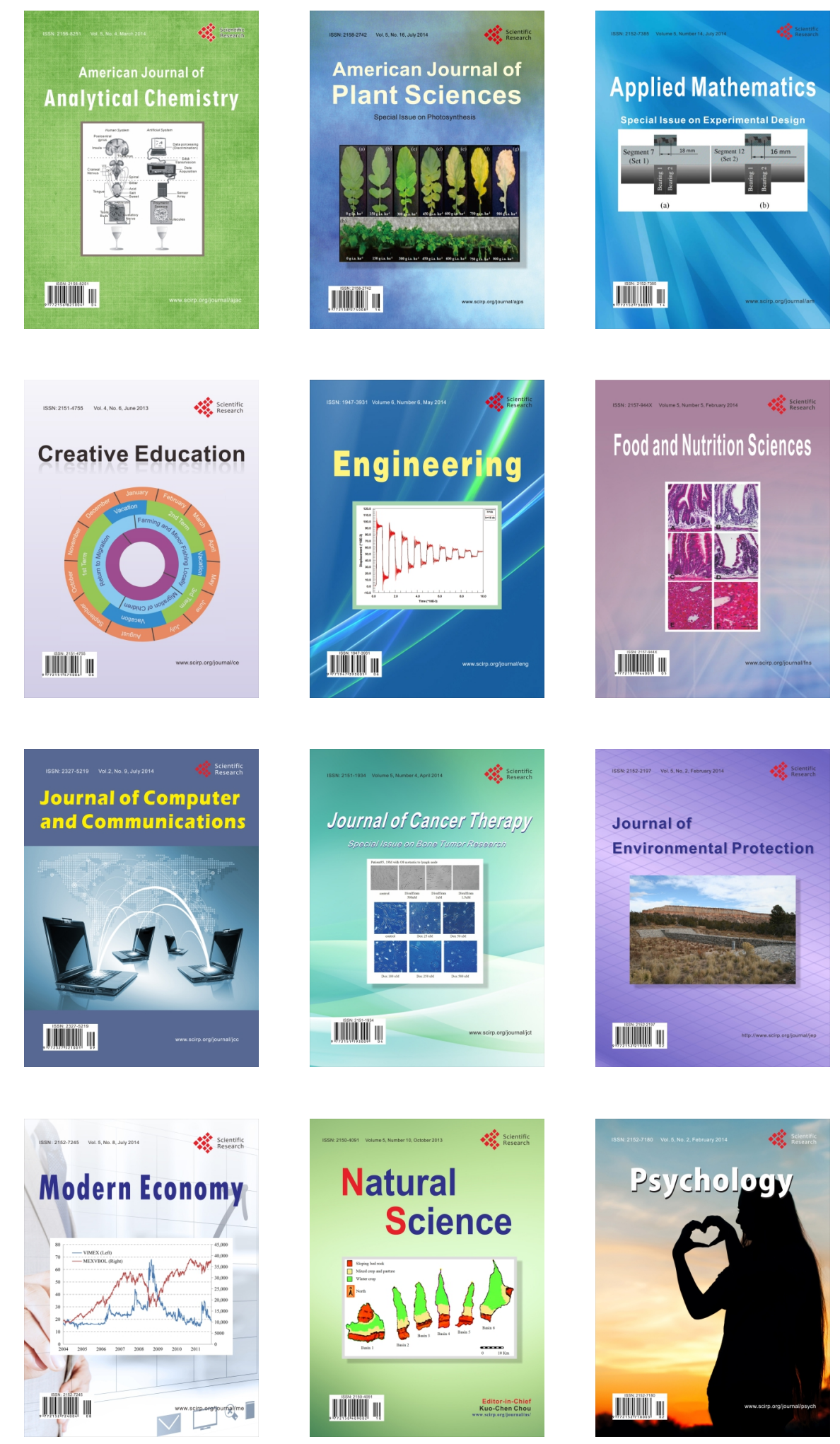